\title{
A Fusion Method of Gabor Wavelet Transform and Unsupervised Clustering Algorithms for Tissue Edge Detection
}

\author{
Burhan Ergen \\ Department of Computer Engineering, Faculty of Engineering, Firat University, 23119 Elazig, Turkey \\ Correspondence should be addressed to Burhan Ergen; burhanergen@gmail.com
}

Received 3 December 2013; Accepted 20 February 2014; Published 23 March 2014

Academic Editors: S. Balochian and V. Bhatnagar

Copyright (C) 2014 Burhan Ergen. This is an open access article distributed under the Creative Commons Attribution License, which permits unrestricted use, distribution, and reproduction in any medium, provided the original work is properly cited.

\begin{abstract}
This paper proposes two edge detection methods for medical images by integrating the advantages of Gabor wavelet transform (GWT) and unsupervised clustering algorithms. The GWT is used to enhance the edge information in an image while suppressing noise. Following this, the $k$-means and Fuzzy $c$-means (FCM) clustering algorithms are used to convert a gray level image into a binary image. The proposed methods are tested using medical images obtained through Computed Tomography (CT) and Magnetic Resonance Imaging (MRI) devices, and a phantom image. The results prove that the proposed methods are successful for edge detection, even in noisy cases.
\end{abstract}

\section{Introduction}

Magnetic Resonance Imaging (MRI) and Computed Tomography $(\mathrm{CT})$ devices are currently the most important diagnostic tools for medical examination. These imaging techniques provide a wealth of information about biological tissues and the condition of anatomical structures. The determination of tissue boundaries plays an important role in many medical applications aimed at identifying the abnormalities in anatomical structures and tissues [1-3]. Also, clinicians planning invasive or noninvasive treatments, such as surgery or radiotherapy, require the correct edge of tumours or tissues to be identified in order to operate. To determine the accurate edge of abnormal tissues helps clinicians to create or modify a treatment plan or to select the region and the path of the operation. Generally, the edge detection and the segmentation are performed by trained radiologists manually. Because the edge detection process is a time consuming task and is subject to radiologist error, researchers have concentrated on developing edge detection methods which detect the accurate edge [4-6]. The detection of the accurate or the acceptable edge is very difficult due to the properties of the analysed image and the variety of modalities.
The medical images received from medical devices, such as MRI or CT, are always corrupted by noise and the artifacts of the devices [7-10]. Therefore, one of the difficulties for edge detection of medical images is the effect of noise and artifacts disturbing the edge of the analyzed images.

It is not enough that detected edges are visually soft and nice. The detected edges should be determined accurately. In the edge detection process, it is commonly considered that an edge detection method should detect all the edges of the objects in the examined image at their correct positions and should not detect non-edge. In an image, the edge is generally obtained using gradient, texture, and intensity, which are the measurable features of the examined image. Early edge detection methods employ local operators to compute the first and second gray-level gradients of an image in the spatial domain. After this, the local maximum locations of the first derivatives or the second derivatives are assumed as the edge points in the analyzed image. In the same way that classical edge detection operators considered benchmark methods [11], Sobel operator, Prewitt operator, LOG operator, and Robert operators compute the derivatives or the gradients. Some edge-detector methods use second-order derivatives like Laplacian or DoG (Difference of Gaussian). If the image 
to be analyzed has poor contrast values and noise between the interested regions and the weak boundaries, the operatorbased edge detection methods encounter difficulties in the detection of the correct edge $[7,8]$.

However a medical image cannot be free of noise and artifacts; the medical images acquired from CT or MRI devices usually suffer from noise and artifacts. These factors reduce the quality of the medical images used to detect the edges. Therefore, a lot of edge detection methods have been put forward recently which detect the boundaries of tissues in a medical image such as wavelet transform, mathematical morphological method, neural networks, or fuzzy methods [12-15].

Even if these studies are successful in identifying the external shape of the interested tissues, it is observed that the usage of the prior information and the shape models does not accurately identify the internal structural changes of the interested tissues. In order to identify the external and internal edge of a tissue, automatic edge detection methods could help because a shape model is not able to model the edge of the internal structures. Thus, some new methods based on soft computing algorithms such as fuzzy, neural, or genetic algorithms have been proposed so that the internal structures may be appropriately determined $[16,17]$.

Since automatic edge detection and segmentation are very difficult, some studies concentrating on a particular problem have used shape models or prior information about the tissues [18]. Although soft computing algorithms have been successful in providing more reliable edges than the traditional and shape modeling methods, it is also reported that these methods are highly sensitive to noise and artifacts $[11,19,20]$. FCM and $k$-means segmentation methods are frequently encountered methods among the soft computing methods used for segmentation problems [21, 22].

Whereas these methods just consider the intensity of the given image, it is reported that FCM cannot give good results if the image is noisy or does not have homogeneous structures $[13,19,23]$. If the existence of noise and artifacts in medical images is taken into consideration, it is clear the FCM is insufficient for the detection of accurate edge. However many algorithms have been proposed for the improvement of FCM; the FCM-based algorithms, when used alone, are still not robust against noise and nonhomogeneity $[19,21$, 24]. The performances of soft computing methods for edge detection are decreased when they are used alone because the noise obscures the weak edges. Traditional edge detectors and soft computing algorithms, which may identify the edge of the internal structures for tissue segmentation, are highly sensitive to noise. If the image has a low signal-to-noise ratio, the traditional edge detectors and the soft computing algorithms fail to determine the contours of the anatomical structures correctly [25].

Therefore, many noise suppression methods have been proposed for the enhancement of the image to be analyzed. However some of the image denoising methods use the pixel relation in spatial domain; the rest assumes that the rapid change in frequency domain refers to noise [26].

While several noise suppression methods which filter the background noise of an image have been proposed, the traditional noise reduction methods are based on the median filter such as Adaptive Weighted Median Filter (AWMF) or the mean filter such as Homogeneous Region Growing Mean Filter (HRGMF) [27]. An improved version of the HRGMF filter, Aggressive Region Growing Filter (ARGF), is proposed in $[28,29]$. This filter uses an adaptive homogeneity threshold instead of the constant threshold value of the HRGMF filter.

Some of the image denoising methods work on frequency domain such as wavelet-based methods. In wavelet-based image denosing process, the image is decomposed into four subimages with respect to their frequency bands in one level decomposition. Afterwards, the small detail coefficients are properly eliminated [26]. Any noise reduction method makes the image blurred less or more. However the noise reduction methods increase the SNR of the given image, and the weak edges in the images become invisible and undetected.

Although edge detection is a very difficult task, humans can easily determine the boundaries within an image without realizing consciously that they are doing so. The human visual system can be modeled as a filter bank. This filter bank can be represented using Gabor functions having different orientation and frequencies. The output of the representation using Gabor function can be accepted as the responses of the human visual system. In particular, the Gabor wavelet transform has demonstrated good performance in texture representation and discrimination $[30,31]$, and it has been successfully applied to face recognition, object detection [32], palm print recognition [33], and also object tracking [34]. Therefore, we have developed a technique integrating the advantages of Gabor wavelet transform and unsupervised clustering algorithms, FCM and $k$-means. The GWT is used as a tool for enhancing the edge of images while suppressing noise. The clustering algorithms convert the gray level edge image into a binary image without any thresholding process. The estimation of an appropriate threshold value is a very difficult task if the histogram of the given image has multiple valleys. Here, we have used the clustering methods, $k$-means and FCM, for the binary conversion avoiding any thresholding process. Figure 1 demonstrates the proposed edge detection method. The proposed algorithm has three steps: the convolution of the input image with Gabor functions, a clustering algorithm to obtain the binary image, and morphological operation to detect the edge.

The paper is organized as follows. Section 2 introduces GWT. In Section 3, $k$-means and the FCM, the most known and used unsupervised algorithms, are presented. In Section 4, the experiments are performed on medical images and a phantom image, in comparison with traditional edge detection methods, Prewitt, Canny, and Sobel. The conclusion is given in Section 5.

\section{Gabor Wavelet Transform (GWT)}

Gabor functions, with different frequencies and orientation, can model the human visual system as a filter bank [33, 35]. A Gabor wavelet can be described as a Gaussian kernel function modulated by a sinusoidal plane wave that has an optimal location in both the frequency domain and the 




FIgURE 1: The proposed edge detection method.

space domain. In literature, there is a significant amount of computer vision applications using Gabor functions, such as texture segmentation, image analysis, and discriminations $[33,36]$.

Gabor wavelets reveal the directional features of an image while providing a fine adjustment for frequency properties $[31,36,37]$. The capability of frequency adjustment is particularly important for the reduction of the background noise in medical images. The preservation of the features of the edge is the most important thing in the noise reduction process. The 2D Gabor wavelet is defined as follows:

$$
\begin{aligned}
& G(x, y, \theta, u, \sigma) \\
& =\frac{1}{2 \pi \sigma^{2}} \exp \left\{-\frac{x^{2}+y^{2}}{2 \sigma^{2}}\right\} \exp \{2 \pi j(u x \cos \theta+u y \sin \theta)\},
\end{aligned}
$$

where $u$ is the frequency of the sinusoidal wave, $\theta$ adjusts the orientation of the wave, $\sigma$ is the standard deviation of the Gaussian function in the $x$ and $y$ direction, and $j=\sqrt{-1}$. The output of the Gabor filtering can be given as a 2D convolution of the input image $I(x, y)$ and $G(x, y)$ for particular $u, \theta$, and $\sigma$. The result is a 2D complex signal because Gabor wavelet is complex. The absolute of this signal is an image preserving the features of the edge. When the wave vector is perpendicular to the edge, the Gabor wavelets enhance the edge and remove the background information. The result image of the convolution demonstrates the local properties indicating the edge of the analyzed image [31]. Kernels related to angles are obtained by setting orientation factor.

Figure 2 shows a different representation for the 2D Gabor wavelet $G(x, y)$ with parameters $\sigma=0.03, u=0.3$ and orientation factor $\theta=0$. Figures 2(a) and 2(c) represent the real part, and Figures 2(b) and 2(d) represent the imaginary part. Actually, Gabor wavelet is a complex wavelet with a few important oscillations relating to frequency parameter. The magnitude decay rate of the oscillations depends on the value of $\sigma$. The characteristics of 2D Gabor wavelet are particularly appropriate to extract the directional features, and the waveform is suitable to preserve the edge pixels while suppressing the noise, which is encountered in medical images.

\section{Unsupervised Clustering}

3.1. K-Means Clustering. A typical unsupervised clustering algorithm is $k$-means which is attractive in practice because it is simple and fast $[38,39]$. The algorithm tries to partition the given input data into $k$ disjoint clusters $c_{i}$. For this purpose, it searches the cluster centers by minimizing the sum of squared distances of each data point $\left(x_{1}, x_{2}, \ldots, x_{N}\right)$ to its nearest cluster centre $c_{j}$. The measure of the distance is commonly chosen as Euclidian distance to minimize the following mean square error (MSE) cost function:

$$
C_{\mathrm{MSE}}=\sum_{i=1}^{k} \sum_{x_{j} \in c_{i}}\left\|x_{j}-c_{i}\right\|^{2},
$$

where $C_{\mathrm{MSE}}$ shows the cost of an examined pixel to assign a cluster, which is the distance.

$x$ and $c$ are the data point and the cluster centre. It can be said that $x$ is in cluster if $\left\|x_{j}-c_{i}\right\|$ is the minimum of all the $k$ distances.

$K$-means algorithm can be summarized as follows.

Step 1. Initializations of centre location $\left(c_{1}, c_{1}, \ldots, c_{k}\right)$.

Step 2. Assigning each $x_{i}$ to its nearest cluster centre $c_{k}$.

Step 3. Deciding the membership of each pixel to the $k$ clusters, whose centroid is closest to that pixel.

Step 4. Setting $c_{i}$ to be the centre of mass of all points in cluster $C_{i}$ for all $k$ cluster centers.

3.2. Fuzzy C-Means Clustering. The clustering process can be expressed as grouping pixels according to the similarities of their features. A clustering algorithm can provide a way of differentiating the regions in an image. Several methods based on the ideas using clustering algorithms have been proposed to partition an image into regions. As an unsupervised technique, Fuzzy c-means (FCM) clustering, was proposed by Bezdek et al. [40] as one of the widely used techniques to determine the boundaries of the objects in an image. It is considered that the reason for the high performance of FCM is due to the fact that through this process each pixel is assigned to a cluster or segment. FCM algorithm groups similar pixels according to their features because an image is represented by its features, such as histogram properties [41].

The cost function, which depends on the distance between the cluster centers and pixels, is calculated iteratively to find a minimum value. The FCM determines the clusters when the cost is minimized. The studies using FCMbased segmentation algorithms reported that FCM-based segmentation methods preserve more information than crisp and hard segmentation methods [42]. However, one of the drawbacks of FCM-based segmentation is sensitivity to noise and imaging artifacts, which is frequently encountered in medical imaging. This disadvantage is due to that fact that spatial information is not taken into account. In FCM, the cluster centers are repositioned after the calculation of an objective function used as $c$-means. There is flexibility in FCM because the objective function includes a membership value to a cluster [24, 41].

In FCM algorithms, each of the pixels is assigned to suitable categories by using a membership function after calculation of a cost function. The cost function is calculated iteratively to find the minimum value using Euclidean 


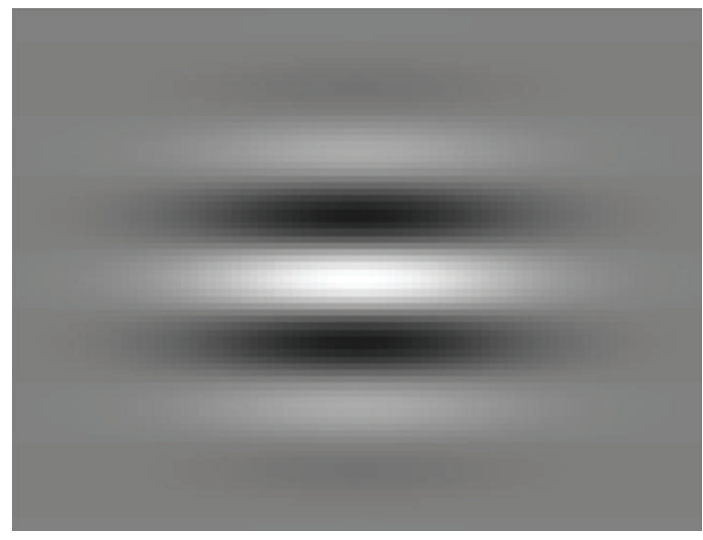

(a)

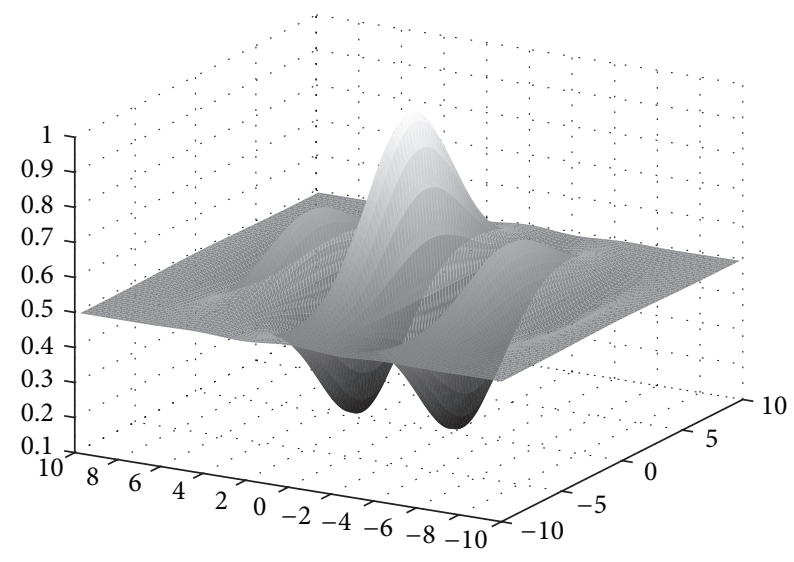

(c)

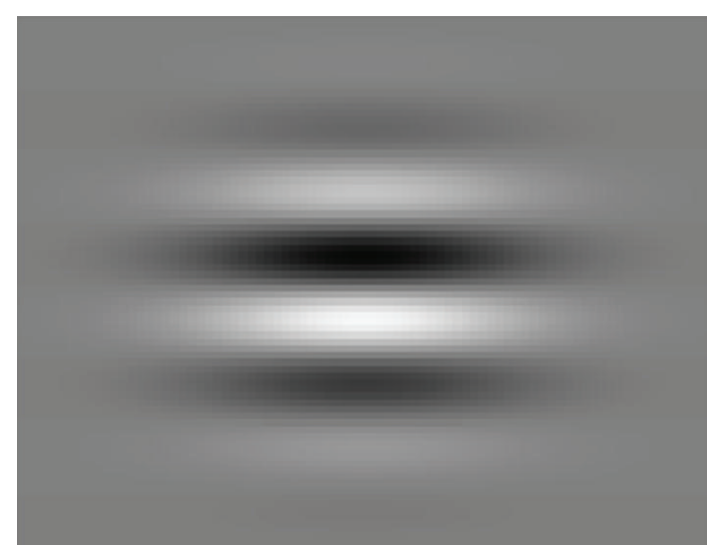

(b)



(d)

Figure 2: A two-dimensional Gabor wavelet; ((a), (c)) real part, ((b), (d)) imaginary part.

distance between the examined pixel and the centre to be assigned. This can be formulated as follows:

$$
E=\sum_{j=1}^{N} \sum_{i=1}^{C} \mu_{i j}^{k}\left\|p_{j}-c_{i}\right\|
$$

where $E$ shows the cost of an examined pixel to assign a cluster. $\mu_{i j}$ and $c_{i}$ represent a membership of a pixel to a cluster and the cluster centre, respectively. $\|\cdot\|$ denotes absolute value operator. $k$ is used to adjust the fuzziness as a constant value.

Here, the membership can be expressed as the probability of a pixel belonging to a cluster. This probability depends on the distance of the pixel to a cluster centre. The probability of the pixel belonging to a cluster can be calculated as follows:

$$
\begin{gathered}
c_{i}=\frac{\sum_{j=1}^{N} \mu_{i j}^{k} x_{j}}{\sum_{j=1}^{N} \mu_{i j}^{k}}, \\
\mu_{i j}=\left(\sum_{m=1}^{C}\left(\frac{\left\|x_{j}-c_{i}\right\|}{\left\|x_{j}-c_{m}\right\|}\right)^{2 /(k-1)}\right)^{-1} .
\end{gathered}
$$

The FCM algorithm process these two equations iteratively.

\section{Experiments}

We have tested our method on several medical images acquired from CT and MRI imaging devices. The edges of the CT brain scan and abdominal images are determined using GWT, an unsupervised clustering algorithm, and morphological skeletonisation. Figure 3 represents a CT brain scan image including a tumour, clustering results, and the tissue edges. The edges are obtained in the three steps in our approach. After applying Gabor wavelet transformation to find out directional edge information, an unsupervised clustering algorithm is used to convert the gray level image into a binary image, which still contains irrelevant pixels. Then, some morphological operations are used to remove the irrelevant pixels in the binary image. As clustering algorithms, we have favored $k$-means and FCM clustering algorithms because they are unsupervised methods. As a morphological method, the skeletonisation is used in order to eliminate the irrelevant pixels in the binary image.

Figure 4 represents the GWT results of the brain image given in Figure 3 using four different orientations $(\pi / 4$, $\pi / 2,3 \pi / 4$, and $\pi$ ). This figure shows how the orientation of GWT plays an important role for the enhancement of the boundaries. The image in Figure 4(e) is the total resulting 


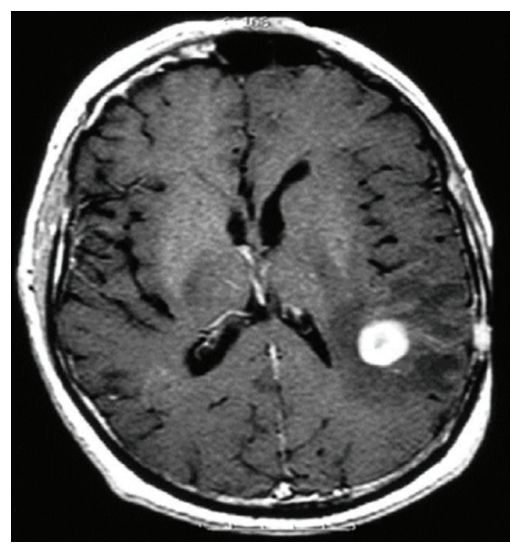

(a)

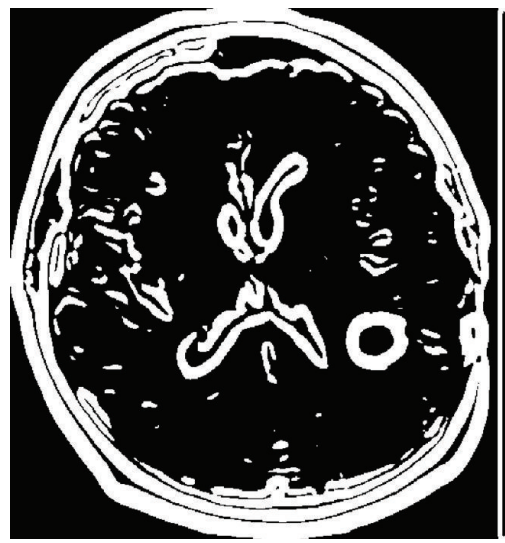

(b)

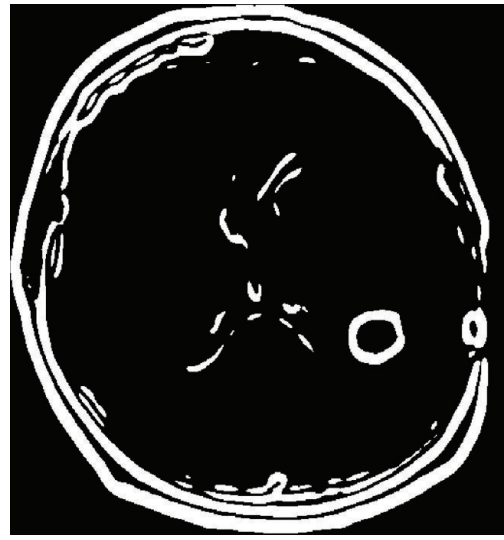

(d)

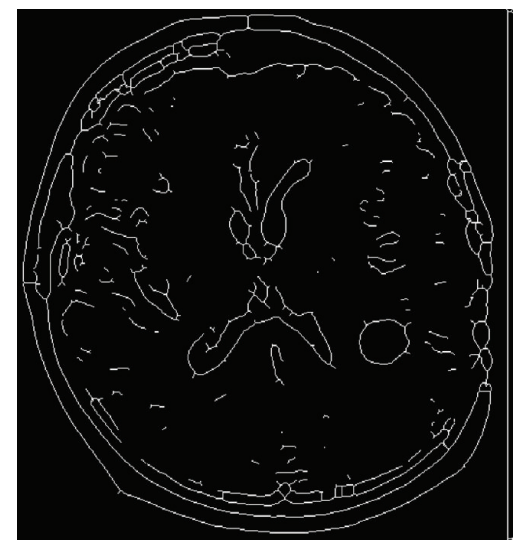

(c)

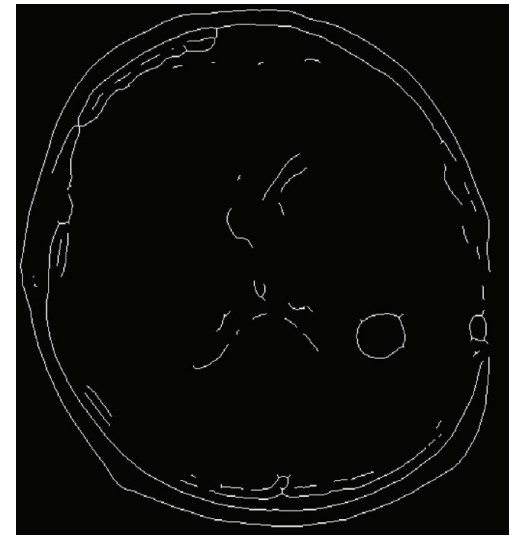

(e)

FIGURE 3: Edges using $k$-means and FCM clustering after GWT; (a) original, (b) $k$-means clustering, (c) skeleton of (b), (d) FCM clustering, and (e) skeleton of (d).

image of the GWT, which contains the total edge information. An unsupervised clustering method is used to convert the gray level image obtained as GWT result into a binary image. Figure 5 represents the binary image obtained using unsupervised methods and their skeletons as an example of abdominal images.

Figures 6 and 7 also represent the results of the proposed method for a CT brain scan image and an abdominal image, respectively. Because the Canny edge detection method is widely used to present the ground truth images in many applications [43], the edge detection results using the Canny method are also given in these figures to carry out a visual comparison.

In fact, it is never possible to identify the accurate edge of a real image. Although there is not a reliable methodology to put forward an appropriate ground truth edge [44], the edge of synthetic image and the manually drawn edge of a real image are used as the ground truth edge. 




(a)



(c)

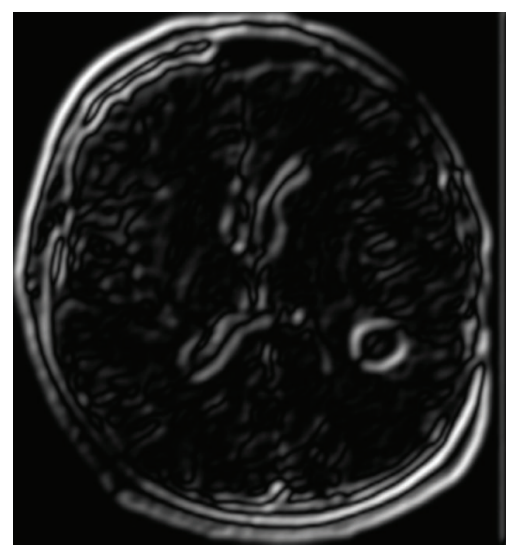

(b)

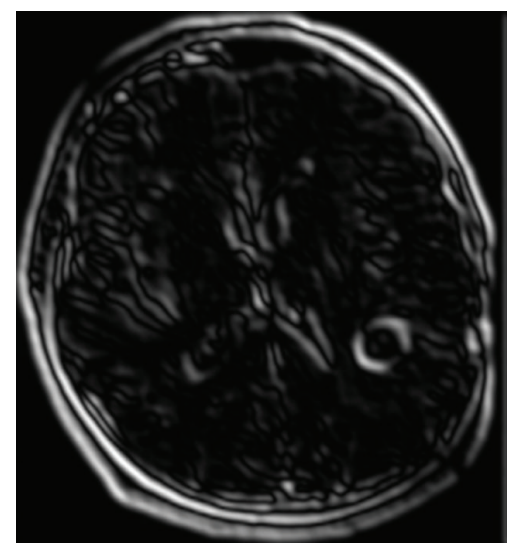

(d)

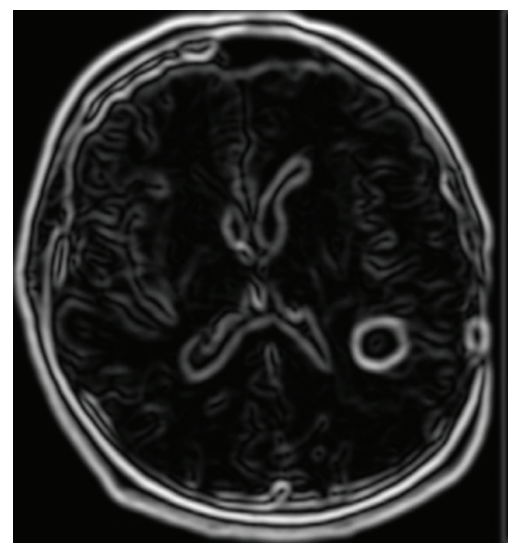

(e)

Figure 4: The GWT of a CT scan brain image for different orientation factors $(\sigma=0.1$ and $\omega=0.005$ ); (a) $\pi / 4$, (b) $\pi / 2$, (c) $3 \pi / 4$, (d) $\pi$, and (e) total result.

Another difficulty is how to define the quality parameter in order to estimate the integrity of edge detection because of application dependency. While several methods have been proposed in the literature to measure the performance of an edge detector objectively, there is no agreement on the quality parameter about edge detection. Nevertheless, the misclassification rate (MCR) and Pratt's figure of Merit (FOM) are proposed to measure the similarity between the ground truth edge and the detected edge in literature.
The MCR can be defined as follows:

$$
\mathrm{MCR}=\frac{\sum\left|B_{A} \cap B_{D}\right|+\sum\left|F_{A} \cap F_{D}\right|}{\sum\left(B_{A}+F_{D}\right)} \times 100 \%,
$$

where $F_{A}$ and $F_{D}$ refer to the foreground pixels of actual and detected image while $B_{A}$ and $B_{D}$ refer to the background pixels of the actual and the detected image. Indeed, the operation of the numerator refers to logical "exor" operation 


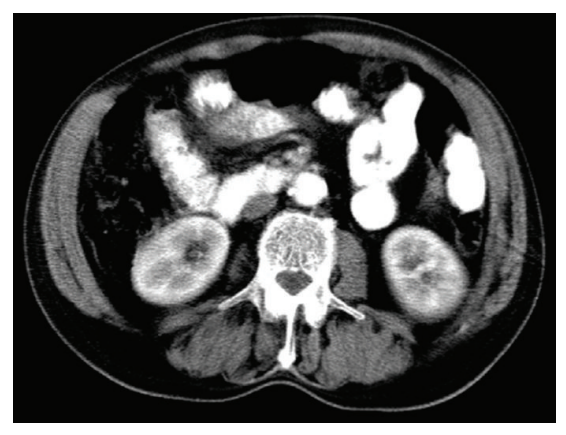

(a)

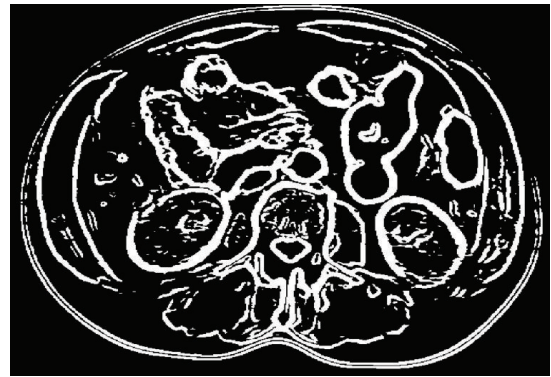

(b)

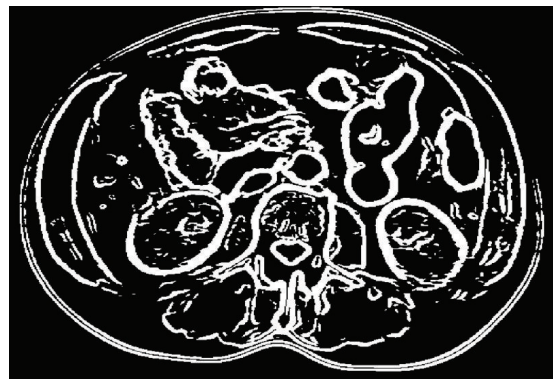

(d)

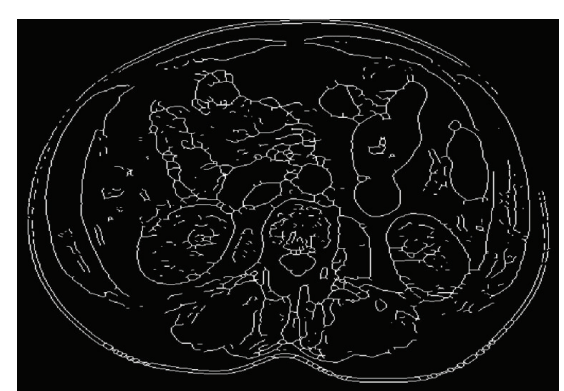

(c)

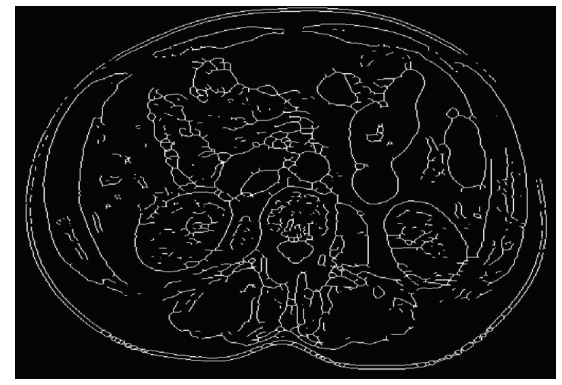

(e)

Figure 5: Edges of an abdominal CT image ( $\sigma=0.18$ and $\omega=0.0005)$; (a) original, (b) $k$-means, (c) skeleton of (b), (d) FCM, (e) skeleton of (d).

$[22,24]$. The less value of MCR indicates that a good detection is done.

The definition of FOM can be given as follows:

$$
\mathrm{FOM}=\frac{1}{\max \left(N_{t}, N_{d}\right)} \sum_{i=1}^{N_{d}} \frac{1}{1+\alpha L(i)^{2}}
$$

where $N$ refers to the number of edge pixels, and subscripts $d$ and $t$ denote the detected edge and the accurate edge, respectively. $L(i)$ shows the distance between the $i$ th accurate edge pixel and the detected edge pixel. And parameter $\alpha$ is generally accepted as $1 / 9$ as a scaling factor [43]. When an accurate edge is not detected, or a false edge is detected, or the detected edge is far from the accurate edge, FOM value increases. If the edge is perfectly detected, FOM value is 1 . Otherwise, it may decrease to zero. The difference between the comparison methods, MCR and FOM, can be expressed as follows. Whereas MCR algorithms accept only the exact overlapped pixel points between the estimated edge and the accurate edge, the FOM algorithms accept the pixel points of the founded edge if they are very near to the accurate edges.

To present the performance of our proposed method, we applied both of the methodologies on a synthetic phantom image and a real image to obtain the appropriate ground truth edges. The images are ordinary CT and MRI images, and no prefiltering procedure is applied. A real and manually annotated approach was performed under the supervision of a radiologist. Figure 8(a) represents the manually ground truth edge of an abdominal image given as an example in Figure 5(a). The edges of the classical methods (Canny, Sobel, and Prewitt), which are accepted as benchmark methods in literature, are also given in Figure 8. Because many edge detection methods are proposed in the literature, we just compared to the most know methods accepted as benchmark.

To evaluate the performance of the proposed method, the MCR and FOM values were calculated using the edge given in Figure 8(a) and other methods, $k$-means, FCM, Prewitt, Canny, and Sobel. Table 1 represents the result of the comparison. These results prove that the performance 


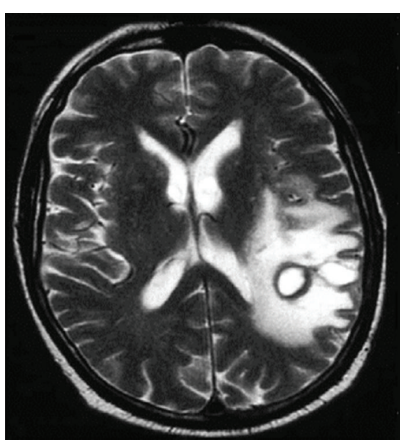

(a)

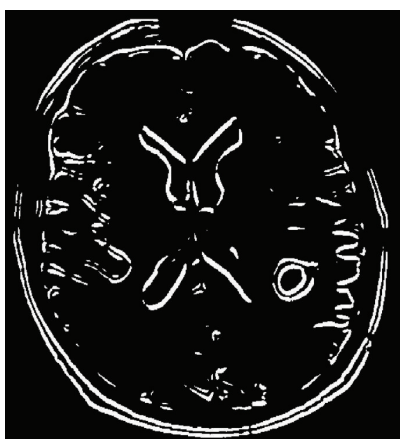

(c)

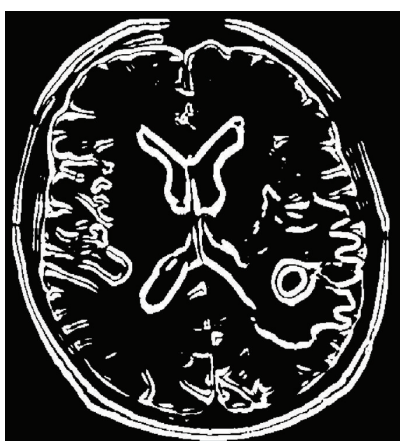

(e)

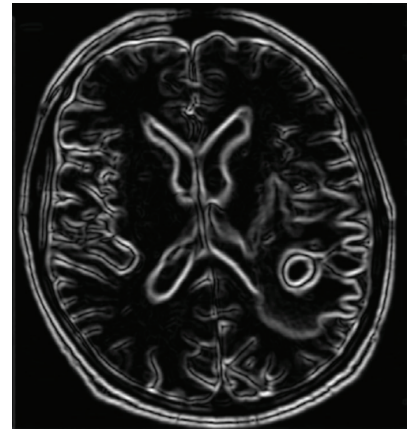

(b)



(d)

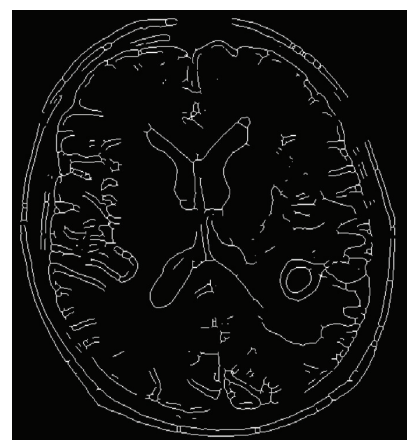

(f)

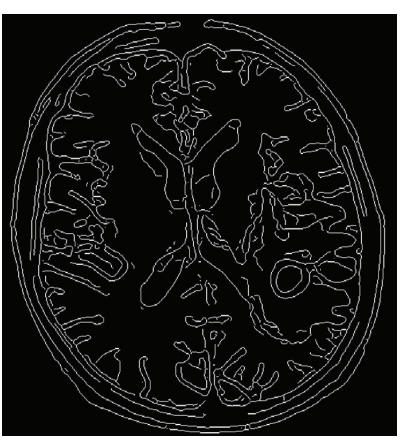

(g)

Figure 6: Edges of a CT scan brain image ( $\sigma=0.3$ and $\omega=0.05$ ); (a) original, (b) GWT, (c) $k$-means, (d) skeleton of (c), (e) FCM, (f) skeleton of (e), and (g) Canny. 


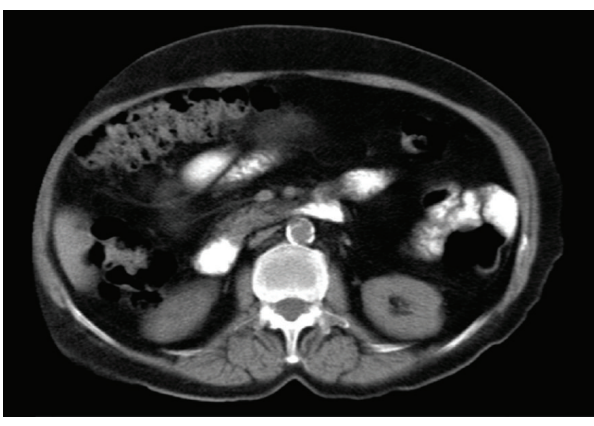

(a)



(c)



(e)

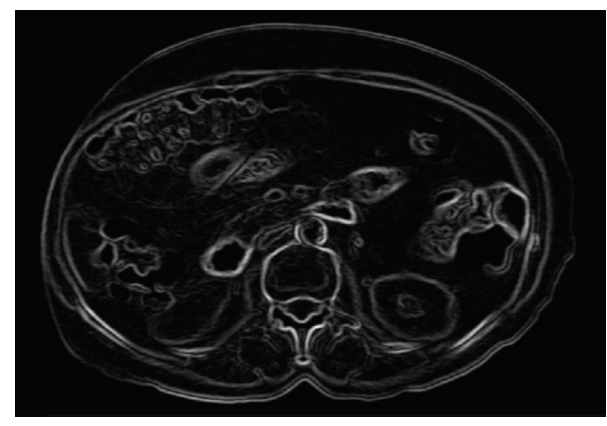

(b)

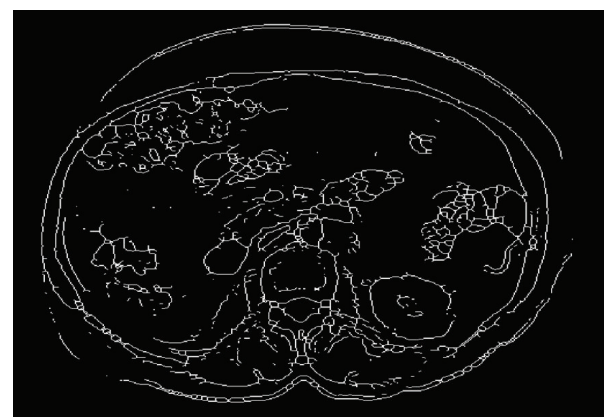

(d)



(f)

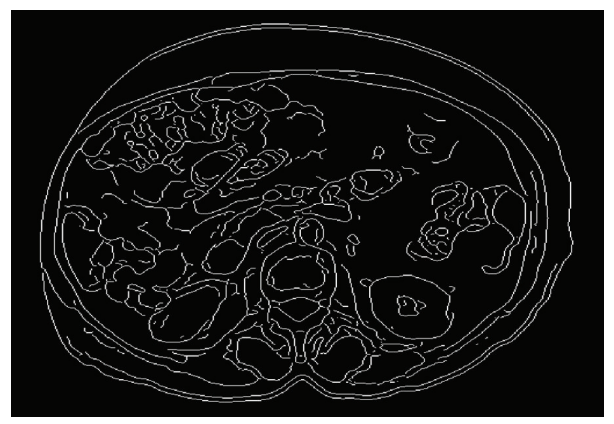

(g)

Figure 7: Edges of a CT scan abdominal image ( $\sigma=0.18$ and $\omega=0.0005)$; (a) original, (b) GWT, (c) $k$-means, (d) skeleton of (c), (d) FCM, (e) skeleton of (d), and (g) Canny.

of the proposed method is higher than the other methods. In particular, FCM represents the highest performance with 0.7981 of FOM values. The Canny edge detection method, which is widely used to obtain a ground truth edge, has the smallest FOM value in the table. The reason for this result is that medical images are always noisy images, and the Canny edge detection method is highly sensitive to noisy signals. $K$-means-based edge detection also has higher FOM value than Canny, Prewitt, and Sobel methods. According to MCR values in Table 1, FCM- and $k$-means-based GWT edge detection methods have smaller values, 4.8653 and 5.6980 , respectively. The fact that MCR results are compatible 


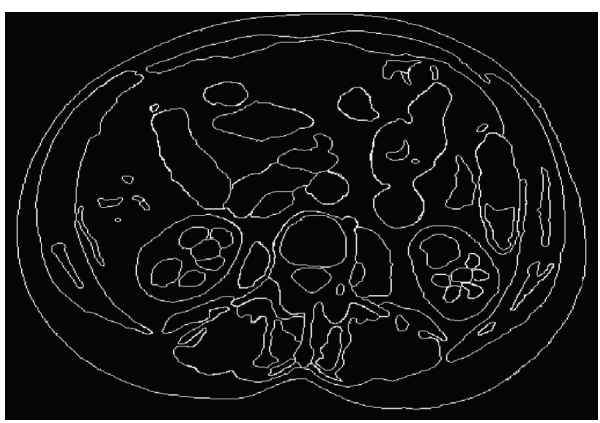

(a)

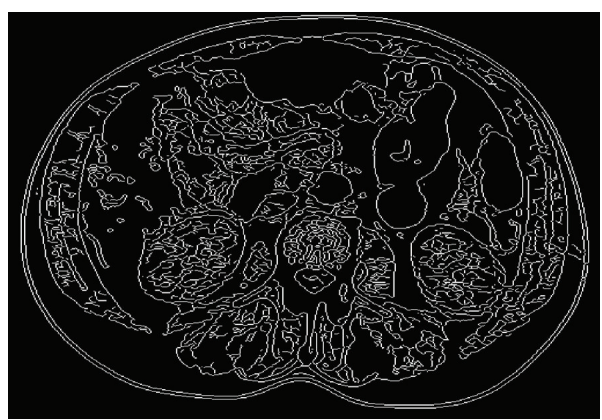

(c)

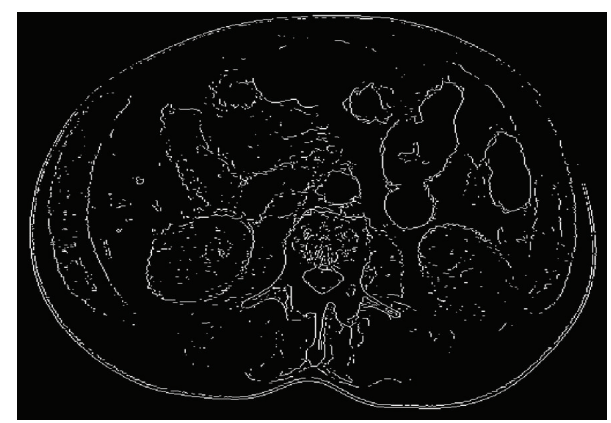

(b)

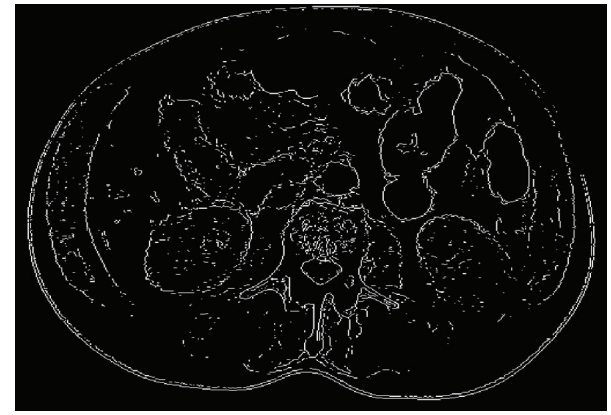

(d)

Figure 8: Edges of Figure 5(a); (a) manually drawn ground truth edge, (b) Prewitt, (c) Canny, and (d) Sobel.

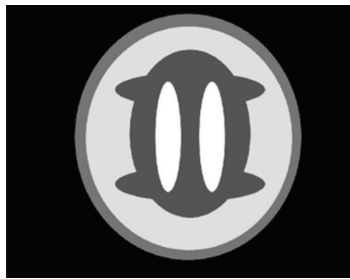

(a)

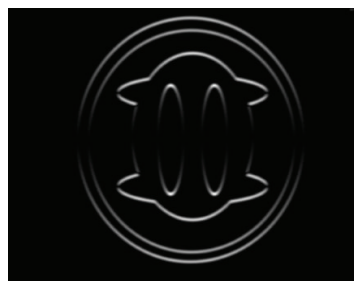

(e)

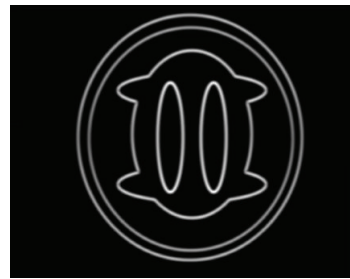

(b)

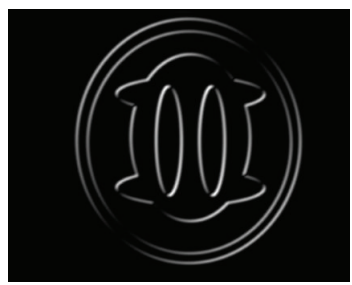

(f)

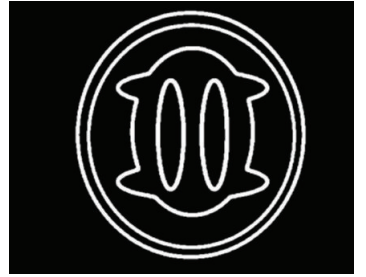

(c)

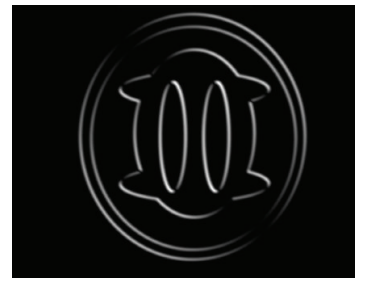

(g)

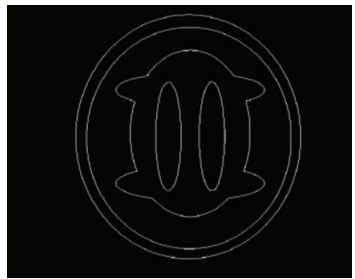

(d)

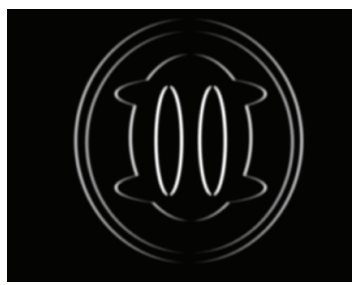

(h)

Figure 9: The simulated image and its GWT ( $\sigma=0.24$ and $\omega=0.0055$ ); (a) original phantom image, (b) GWT, (c) $k$-means, (d) skeleton of (c), (e) $\pi / 4$, (f) $\pi / 2$, (g) $3 \pi / 4$, and (h) $\pi$.

with FOM results supports that GWT-based edge detection methods have higher performances.

As stated, it is very difficult to find the edge of a medical image corrupted by noisy signals. Medical images are generally low-density and noisy images depending on the type of imaging device. Furthermore, no medical imaging device can operate independently of the noise. Therefore, the resistance of an edge detection method to noise should be taken into consideration, particularly in medical imaging.
In order to measure the resistance to noisy signals, we have performed another experiment using a synthetic phantom image given in Figure 9(a). Why we use the phantom image and its noisy form is to make a more objective assessment.

The phantom image is constituted using several overlapping ellipses. It is assumed each ellipse indicates to a tissue. The accurate edge accepted as the ground truth edge was determined when the phantom image was constituted. The MCR and FOM values are computed for the phantom image 
TABLE 1: MCR and FOM results for the manually ground truth edge in Figure 8(a).

\begin{tabular}{lccccccccc}
\hline & \multicolumn{3}{c}{ MCR results } & & \multicolumn{3}{c}{ FOM results } \\
\hline 5.6980 & 4.8653 & 6.1733 & 9.3037 & 6.2557 & 0.7195 & 0.7981 & 0.7010 & 0.5020 & 0.7094 \\
\hline
\end{tabular}

TABLE 2: MCR and FOM results for the phantom image in Figure 9.

\begin{tabular}{|c|c|c|c|c|c|c|c|c|c|c|}
\hline \multirow{2}{*}{ PSNR } & \multicolumn{5}{|c|}{ MCR results } & \multicolumn{5}{|c|}{ FOM results } \\
\hline & $k$-means & $\mathrm{FCM}$ & Prewitt & Canny & Sobel & $k$-means & FCM & Prewitt & Canny & Sobel \\
\hline 35.01 & 0.9384 & 0.9285 & 0.8934 & 0.7954 & 0.8968 & 0.9710 & 0.9710 & 0.9723 & 0.9752 & 0.9722 \\
\hline 30.01 & 0.9182 & 0.9350 & 0.9007 & 16.8981 & 0.8938 & 0.9712 & 0.9709 & 0.9721 & 0.1902 & 0.9723 \\
\hline 25.02 & 0.9548 & 0.9563 & 0.9102 & 18.1576 & 0.9243 & 0.9700 & 0.9701 & 0.9614 & 0.0765 & 0.9622 \\
\hline 23.00 & 1.2249 & 1.3115 & 2.9491 & 21.2528 & 2.9976 & 0.9429 & 0.8958 & 0.4788 & 0.0658 & 0.4690 \\
\hline $23.00^{*}$ & 1.2196 & 1.3355 & 2.0824 & 7.6271 & 2.0962 & 0.9351 & 0.8985 & 0.7296 & 0.1831 & 0.7236 \\
\hline
\end{tabular}

"Preprocessed using median filter.

to conclude an evaluation in order to measure the performance of edge detection methods on the phantom image. The results given in Table 2 show that the proposed algorithm can detect the edges more precisely than the classical method even in the presence of noise. It can be considered that better results have been yielded by the proposed method because the GWT acts as a gradient operator while suppressing noise. The last row $(*)$ shows the results of the edge detection methods after applying a median filter on the noisy phantom image. The filter operation is applied to the noisy phantom image only when using classical methods. No filter operation is applied to the phantom image when using the GWT-based methods. Even in this case, the proposed methods give higher FOM values and smaller MCR values, as seen in Table 2. This last result proves that GWT is successful even in noisy cases.

The FOM values of the proposed method may change slightly with respect to the trail number because $k$-means and FCM clustering methods choose the centroid arbitrarily. According to the results of the phantom image, it is observed that the FCM clustering algorithm is more sensitive than the $k$-means clustering algorithm. Nonetheless, the proposed method using $k$-means and FCM is very successful compared to other methods.

\section{Conclusion}

This work presents two methods of edge detection based on the GWT. These two proposed methods use $k$-means and FCM clustering method to convert a gray level image into a binary image. The main idea of the proposed method is to integrate the information obtained from the GWT at a different orientation and to incorporate the use of a clustering method. The effect of the GWT can be seen on the regional boundaries of the given image. The GWT enhances the edge information and suppresses the noisy signals in a given image. The tests prove that both methods have a great performance particularly in noisy conditions.

In this paper, we have proposed two kinds of edge detection methods based on GWT. Other methods using GWT could be conceptually explored and adapted. The results showed that the directional information from GWT provides a competitive advantage for edge analysis and detection. Since GWT uses three parameters, sigma, frequency, and orientation, it can be adapted for application-dependent images.

\section{Conflict of Interests}

The author wishes to confirm that there is no known conflict of interests associated with this paper and there has been no significant financial support for this work that could have influenced its outcome. The author confirms that the paper has been read and approved and that there are no other persons who satisfied the criteria for authorship and are not listed.

\section{Acknowledgment}

The author would like to thank Dr. Murat Baykara for sharing medical images and for giving advice. The author also confirms that he has given due consideration to the protection of intellectual property associated with this work and that there are no impediments to publication, including the timing of publication, with respect to intellectual property. In doing so, the author confirms that he has followed the regulations of his institution concerning intellectual property. He further confirms that any aspect of the work covered in this paper that has involved either experimental animals or human patients has been conducted with the ethical approval of all relevant bodies and that such approvals are acknowledged within the paper. The author understands that the corresponding author is the sole contact for the editorial process (including editorial manager and direct communications with the office). He/she is responsible for communicating with the other authors about progress, submissions of revisions, and final approval of proofs. The author confirms that he has provided a current, correct email address which is accessible by the corresponding author. 


\section{References}

[1] Z. Ma, J. M. R. S. Tavares, R. N. Jorge, and T. Mascarenhas, "A review of algorithms for medical image segmentation and their applications to the female pelvic cavity," Computer Methods in Biomechanics and Biomedical Engineering, vol. 13, no. 2, pp. 235246, 2010.

[2] R.-R. Jorge and B.-C. Eduardo, "Medical image segmentation, volume representation and registration using spheres in the geometric algebra framework," Pattern Recognition, vol. 40, no. 1, pp. 171-188, 2007.

[3] Q. Abbas, M. E. Celebi, and I. F. Garćra, "Breast mass segmentation using region-based and edge-based methods in a 4-stage multiscale system," Biomedical Signal Processing and Control, vol. 8, no. 2, pp. 204-214, 2013.

[4] X. Lu, Y. Sun, and Y. Yuan, "Optimization for limited angle tomography in medical image processing," Pattern Recognition, vol. 44, no. 10-11, pp. 2427-2435, 2011.

[5] Z. Zhang, S. Ma, H. Liu, and Y. Gong, "An edge detection approach based on directional wavelet transform," Computers and Mathematics with Applications, vol. 57, no. 8, pp. 1265-1271, 2009.

[6] A. Fathi and A. R. Naghsh-Nilchi, "Automatic wavelet-based retinal blood vessels segmentation and vessel diameter estimation," Biomedical Signal Processing and Control, vol. 8, no. 6, pp. 71-80, 2013.

[7] P. Coupé, J. V. Manjón, E. Gedamu, D. Arnold, M. Robles, and D. L. Collins, "Robust Rician noise estimation for MR images," Medical Image Analysis, vol. 14, no. 4, pp. 483-493, 2010.

[8] M. Ikeda, R. Makino, K. Imai, M. Matsumoto, and R. Hitomi, "A method for estimating noise variance of CT image," Computerized Medical Imaging and Graphics, vol. 34, no. 8, pp. 642-650, 2010.

[9] Y. Dai and G. L. Niebur, "A semi-automated method for hexahedral mesh construction of human vertebrae from CT scans," Computer Methods in Biomechanics and Biomedical Engineering, vol. 12, no. 5, pp. 599-606, 2009.

[10] H. Masoumi, A. Behrad, M. A. Pourmina, and A. Roosta, "Automatic liver segmentation in MRI images using an iterative watershed algorithm and artificial neural network," Biomedical Signal Processing and Control, vol. 7, no. 5, pp. 429-437, 2012.

[11] Q. He and Z. Zhang, "A new edge detection algorithm for image corrupted by White-Gaussian noise," AEU-International Journal of Electronics and Communications, vol. 61, no. 8, pp. 546550, 2007.

[12] Z. Dokur and T. Ölmez, "Tissue segmentation in ultrasound images by using genetic algorithms," Expert Systems with Applications, vol. 34, no. 4, pp. 2739-2746, 2008.

[13] Z.-X. Ji, Q.-S. Sun, and D.-S. Xia, "A framework with modified fast FCM for brain MR images segmentation," Pattern Recognition, vol. 44, no. 5, pp. 999-1013, 2011.

[14] T. Chen, Q. H. Wu, R. Rahmani-Torkaman, and J. Hughes, "A pseudo top-hat mathematical morphological approach to edge detection in dark regions," Pattern Recognition, vol. 35, no. 1, pp. 199-210, 2002.

[15] S. Anand, R. S. S. Kumari, S. Jeeva, and T. Thivya, "Directionlet transform based sharpening and enhancement of mammographic X-ray images," Biomedical Signal Processing and Control, vol. 8, pp. 391-399, 2013.

[16] J.-Y. Zhang, C. Yan, and X.-X. Huang, "Edge detection of images based on improved sobel operator and genetic algorithms," in
Proceedings of the International Conference on Image Analysis and Signal Processing (IASP '09), pp. 31-35, April 2009.

[17] Z. Iscan, A. Yüksel, Z. Dokur, M. Korürek, and T. Ölmez, "Medical image segmentation with transform and moment based features and incremental supervised neural network," Digital Signal Processing, vol. 19, no. 5, pp. 890-901, 2009.

[18] V. Grau, A. U. J. Mewes, M. Alcañiz, R. Kikinis, and S. K. Warfield, "Improved watershed transform for medical image segmentation using prior information," IEEE Transactions on Medical Imaging, vol. 23, no. 4, pp. 447-458, 2004.

[19] M. A. Balafar, A. R. Ramli, M. I. Saripan, and S. Mashohor, "Medical image segmentation using fuzzy C-mean (FCM), bayesian method and user interaction," in Proceedings of the International Conference on Wavelet Analysis and Pattern Recognition (ICWAPR '08), pp. 68-73, August 2008.

[20] T. Cerciello, P. Bifulco, M. Cesarelli, and A. Fratini, "A comparison of denoising methods for X-ray fluoroscopic images," Biomedical Signal Processing and Control, vol. 7, no. 6, pp. 550559, 2012.

[21] W. Cai, S. Chen, and D. Zhang, "Fast and robust fuzzy c-means clustering algorithms incorporating local information for image segmentation," Pattern Recognition, vol. 40, no. 3, pp. 825-838, 2007.

[22] K.-S. Chuang, H.-L. Tzeng, S. Chen, J. Wu, and T.-J. Chen, "Fuzzy c-means clustering with spatial information for image segmentation," Computerized Medical Imaging and Graphics, vol. 30, no. 1, pp. 9-15, 2006.

[23] L. O. Hall, A. M. Bensaid, L. P. Clarke, R. P. Velthuizen, M. S. Silbiger, and J. C. Bezdek, "A comparison of neural network and fuzzy clustering techniques in segmenting magnetic resonance images of the brain," IEEE Transactions on Neural Networks, vol. 3, no. 5, pp. 672-682, 1992.

[24] T. Chaira, "A novel intuitionistic fuzzy C means clustering algorithm and its application to medical images," Applied Soft Computing Journal, vol. 11, no. 2, pp. 1711-1717, 2011.

[25] L. Zhang and P. Bao, "Edge detection by scale multiplication in wavelet domain," Pattern Recognition Letters, vol. 23, no. 14, pp. 1771-1784, 2002.

[26] B. Ergen, "Signal and image denoising using wavelet transform," in Advances in Wavelet Theory and Their Applications in Engineering, Physics and Technology, D. Baleanu, Ed., pp. 495-514, 2012.

[27] A. Thakur and R. S. Anand, "Speckle reduction in ultrasound medical images using adaptive filter based on second order statistics," Journal of Medical Engineering and Technology, vol. 31, no. 4, pp. 263-279, 2007.

[28] Y. Yue, M. M. Croitoru, A. Bidani, J. B. Zwischenberger, and J. W. Clark Jr., "Nonlinear multiscale wavelet diffusion for speckle suppression and edge enhancement in ultrasound images," IEEE Transactions on Medical Imaging, vol. 25, no. 3, pp. 297-311, 2006.

[29] C. Zhu, J. Ni, Y. Li, and G. Gu, "Speckle noise suppression techniques for ultrasound images," in Proceedings of the 4th International Conference on Internet Computing for Science and Engineering (ICICSE '09), pp. 122-125, December 2009.

[30] W. D. Richard and C. G. Keen, "Automated texture-based segmentation of ultrasound images of the prostate," Computerized Medical Imaging and Graphics, vol. 20, no. 3, pp. 131-140, 1996.

[31] W. Jiang, K.-M. Lam, and T.-Z. Shen, "Efficient edge detection using simplified Gabor wavelets," IEEE Transactions on Systems, Man, and Cybernetics B: Cybernetics, vol. 39, no. 4, pp. 10361047, 2009. 
[32] L. Shen and L. Bai, "A review on Gabor wavelets for face recognition," Pattern Analysis and Applications, vol. 9, no. 2-3, pp. 273-292, 2006.

[33] X. Pan and Q.-Q. Ruan, "Palmprint recognition using Gaborbased local invariant features," Neurocomputing, vol. 72, no. 7-9, pp. 2040-2045, 2009.

[34] "Kernel based multi-object tracking using gabor functions embedded in a region covariance matrix," in Pattern Recognition and Image Analysis, vol. 5524 of Lecture Notes in Computer Science, pp. 72-79, 2009.

[35] D. Shen, Y. Zhan, and C. Davatzikos, "Segmentation of prostate boundaries from ultrasound images using statistical shape model," IEEE Transactions on Medical Imaging, vol. 22, no. 4, pp. 539-551, 2003.

[36] W. Gu, C. Xiang, Y. V. Venkatesh, D. Huang, and H. Lin, "Facial expression recognition using radial encoding of local Gabor features and classifier synthesis," Pattern Recognition, vol. 45, no. 1, pp. 80-91, 2012.

[37] J. V. B. Soares, J. J. G. Leandro, R. M. Cesar Jr., H. F. Jelinek, and M. J. Cree, "Retinal vessel segmentation using the 2-D Gabor wavelet and supervised classification," IEEE Transactions on Medical Imaging, vol. 25, no. 9, pp. 1214-1222, 2006.

[38] K. R. Žalik, "An efficient $\mathrm{k}^{\prime}$-means clustering algorithm," Pattern Recognition Letters, vol. 29, no. 9, pp. 1385-1391, 2008.

[39] P. J. Herrera, G. Pajares, and M. Guijarro, "A segmentation method using Otsu and fuzzy k-Means for stereovision matching in hemispherical images from forest environments," Applied Soft Computing Journal, vol. 11, no. 8, pp. 4738-4747, 2011.

[40] J. C. Bezdek, R. Ehrlich, and W. Full, "FCM: the fuzzy c-means clustering algorithm," Computers and Geosciences, vol. 10, no. 23, pp. 191-203, 1984.

[41] Z. Ji, Y. Xia, Q. Chen, Q. Sun, D. Xia, and D. D. Feng, "Fuzzy c-means clustering with weighted image patch for image segmentation," Applied Soft Computing Journal, vol. 12, no. 6, pp. 1659-1667, 2012.

[42] Y. Li and G. Li, "Fuzzy C-means cluster segmentation algorithm based on modified membership," in Advances in Neural Networks: ISNN 2009, vol. 5552 of Lecture Notes in Computer Science, pp. 135-144, 2009.

[43] S. Yi, D. Labate, G. R. Easley, and H. Krim, "A shearlet approach to edge analysis and detection," IEEE Transactions on Image Processing, vol. 18, no. 5, pp. 929-941, 2009.

[44] N. L. Fernández-García, A. Carmona-Poyato, R. MedinaCarnicer, and F. J. Madrid-Cuevas, "Automatic generation of consensus ground truth for the comparison of edge detection techniques," Image and Vision Computing, vol. 26, no. 4, pp. 496-511, 2008. 

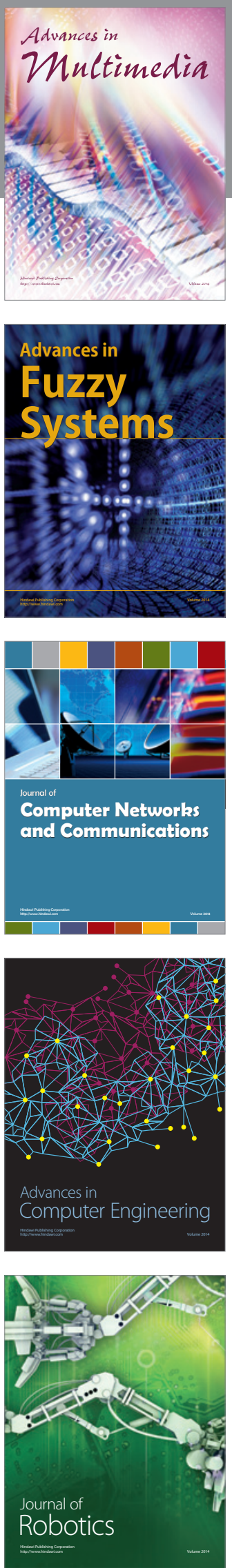

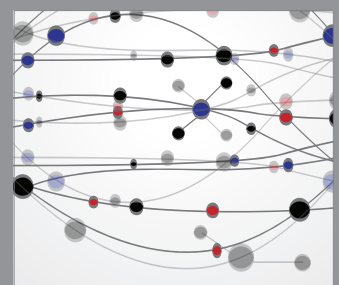

The Scientific World Journal


Submit your manuscripts at

http://www.hindawi.com
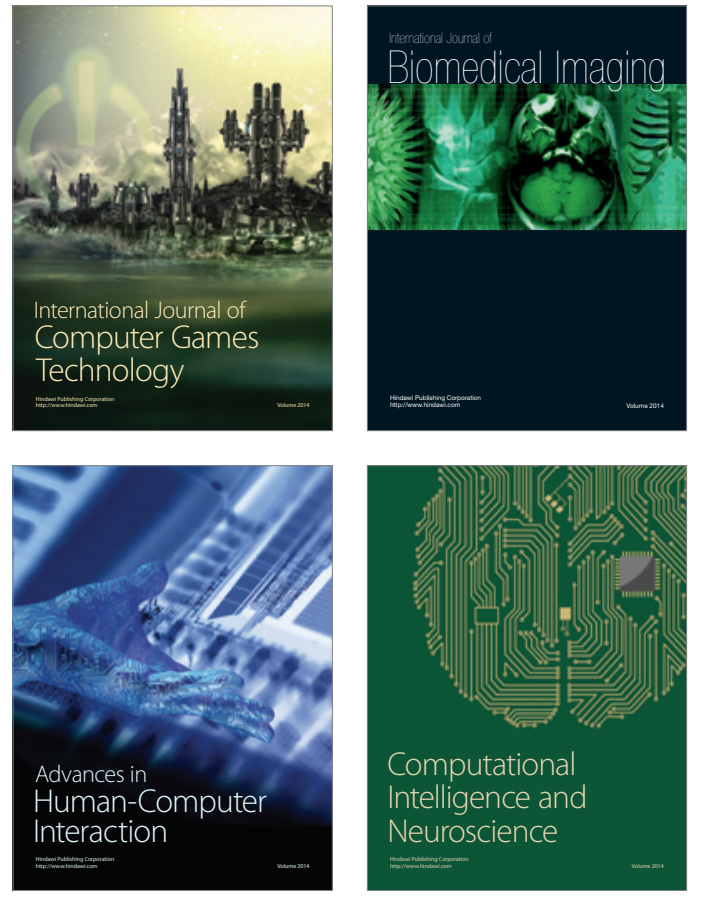
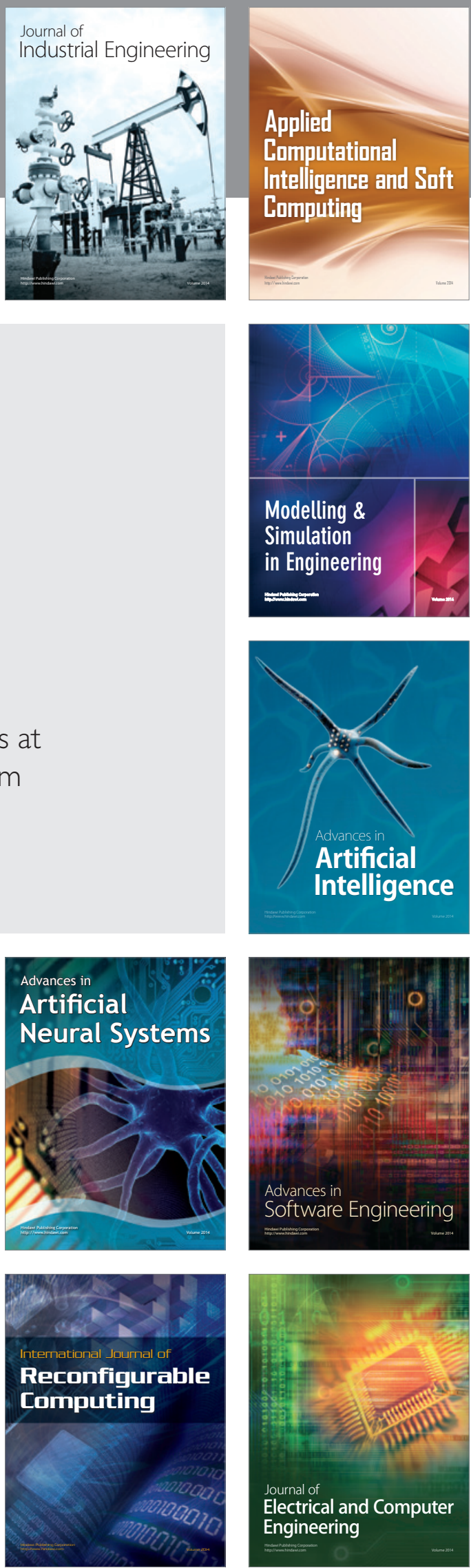